\title{
Grupo focal con nutricionistas que actúan \\ en la alimentación escolar: el menú como elemento pedagógico
}

\author{
Focus group with nutritionists working \\ in school food: the menu as \\ a teaching element
}

\begin{abstract}
The debate over school feeding (SF) and its impact on the epidemiological profile requires the formulation of regulatory actions and educational planning. This study aimed to discuss the menu management and recognition in the educational aspect of the SF. This was a qualitative study involving nutritionists working at the SF network of neighboring municipalities of the Greater São Paulo, whose data collection used focus group and analysis followed the approach of thematic networks. At the moment to mention the planned educational strategies, the participants emphasized not having done as desired due to overwork and although the menu management is of great concern, it is not recognized as an educational tool. The interpretation of education can be recognized as formal and explicit actions; however, menu management transcends traditional communication channels, by focusing on consumption as a time of learning and promoting healthy eating habits, contributing and reinforcing the prevention of obesity at the school environment.
\end{abstract}

Key words: School feeding; menu planning; nutrition programs; school health; obesity.

\section{INTRODUCCIÓN}

El perfil epidemiológico actual de niños y adolescentes ha provocado debate en torno a la alimentación escolar (AE), lo que compromete tanto intencionalidad como planeación educativa. La mayor incidencia de obesidad infantil está íntimamente asociada al estilo de vida, incluyendo sedentarismo y consumo bajo de verduras, frutas, derivados lácteos y alto de alimentos procesados. Esas características son antagónicas a lo recomendado, es decir, la alimentación de este grupo poblacional, inclusive en el propio contexto escolar, debería basarse en la oferta variada de alimentos que permita asegurar un estado nutricional adecuado y estimular hábitos alimentarios saludables para que perduren a lo largo de la vida (1).

De acuerdo con Kac \& Velásquez-Meléndez (2) es indudable que Brasil y otros países de América Latina están experimentando en los últimos años una rápida transición demográfica, epidemiológica y nutricional, y dentro de ese contexto, la obesidad se consolida como una complicación asociada a la alta incidencia de enfermedades cardiovasculares, cáncer y diabetes, influyendo de esta manera en el perfil de morbimortalidad de las poblaciones. Estos hechos señalan la
Ana Maria Cervato-Mancuso Gabriela Moraes da Silva Alexandra Pava Cárdenas

Facultad de Salud Pública, Universidad de San Pablo, San Pablo, Brasil

$$
\begin{array}{r}
\text { Dirigir la correspondencia a: } \\
\text { Profesora } \\
\text { Ana María Cervato-Mancuso } \\
\text { Universidad de San Pablo, Facultad de Salud Pública } \\
\text { O1246-904 - San Pablo } \\
\text { SP - Brasil } \\
\text { Fax: (11) 30617736 } \\
\text { E-mail: cervato@usp.br }
\end{array}
$$

Este trabajo fue recibido el 26 de Marzo de 2013 y aceptado para ser publicado el 10 de Agosto de 2013.

importancia de analizar la efectividad de distintas acciones de promoción de la salud dirigidas a toda la población y especialmente en el escenario de la escuela, con el fin de elaborar nuevas estrategias para ser desarrolladas en el área de $A E$, haciendo frente a este problema de salud pública (3).

En el caso particular de Brasil, el Programa Nacional de Alimentación Escolar (PNAE) implementado en 1955 se destaca como la primera iniciativa pública formal en dicha área. Mediante la transferencia de recursos financieros, este programa garantiza la AE de los estudiantes de educación básica (educación primaria y secundaria, incluyendo jóvenes y adultos) matriculados en escuelas públicas y filantrópicas (4).

El objetivo del PNAE es atender las necesidades nutricionales de los alumnos durante su permanencia en las aulas, contribuyendo al crecimiento, desarrollo, aprendizaje y rendimiento escolar, así como la formación de hábitos alimentarios saludables. El PNAE es considerado uno de los mayores programas en el área de alimentación escolar del mundo y es el único con una atención integral. Su alta cobertura ofrece una gran oportunidad para la inserción de la Educación Alimentaria y Nutricional (EAN) en las escuelas. 
De acuerdo con Salinas C. et al. (5), como consecuencia de la rápida transición epidemiológica y nutricional, los procesos de cambio en las políticas de educación y salud ofrecen nuevas oportunidades para el desarrollo de estrategias integradas en las escuelas, que deben responder de forma eficaz a la situación de salud y nutrición de preescolares y escolares.

Como indica Iuliano (6), la escuela es un ambiente favorable para desarrollar estrategias de EAN, ya que además de atender a los escolares, puede involucrar a la familia y sociedad. Sumado a todo esto, el Fondo Internacional de Emergencia de las Naciones Unidas para la Infancia (UNICEF) refiere que las intervenciones en las escuelas representan una de las mejores relaciones costo-efectividad y son medios sostenibles para promover prácticas saludables.

El estudio realizado en Guarulhos, municipio perteneciente a la región metropolitana de San Pablo (7), muestra las potenciales posibilidades educativas en el ámbito escolar y su inserción temática en el currículo. Por medio del acompañamiento a $20 \%$ de las unidades activas de educación básica municipal, se programaron actividades relacionadas con alimentación, previstas en los proyectos político-pedagógicos, aunque sin la participación de los nutricionistas del PNAE. Se identificaron algunas estrategias educativas realizadas durante el consumo de alimentos o dirigidas a ese momento pedagógico, como las orientaciones informales del profesor y del funcionario del servicio, teniendo como asuntos principales: "la alimentación equilibrada con relación al valor nutricional" y "la salud". Además hubo clases prácticas, incluyendo la huerta que fue la más frecuentada, en las que se desarrollo EAN con mayor complejidad.

El profesional encargado de la AE es el nutricionista, quien responde por los aspectos éticos, civiles, administrativos y penales. Dicha responsabilidad técnica es compatible con su formación y principios éticos que buscan calidad en los servicios prestados a la sociedad (8). En 2009, buscando establecer una nueva reglamentación para el programa, entró en vigencia una ley sobre la atención de la alimentación escolar (9) que recoge de una manera explícita el derecho a la alimentación escolar y el derecho humano a la alimentación adecuada e incluye la EAN en el proceso de enseñanza y aprendizaje, representando un nuevo desafío para la formación profesional en el campo de la AE.

Teniendo en cuenta que la oferta y la disponibilidad de una alimentación saludable son herramientas fundamentales para la promoción de prácticas alimentarias saludables, este trabajo tuvo como objetivo discutir la gestión del menú y el reconocimiento de su vertiente educacional en la $\mathrm{AE}$.

\section{SUJETOS Y MÉTODOS}

La Región Metropolitana de San Pablo está constituida por 39 municipios, dentro de los cuales se contemplaron San Pablo y seis municipios próximos a este: Osasco, Cotia, Suzano, São Bernardo do Campo, Embu das Artes y Guarulhos. En dicha microrregión hay 2.508 escuelas estatales y municipales con jardín, escuela primaria y secundaria. En el programa de Alimentación Escolar Nacional aparece registrados 7000 nutricionistas, sin embargo la asignación de nutricionistas es por municipio y es este el encargado de adjudicar las escuelas (10).

El estudio desarrollado siguió la línea de abordaje cualitativa, dado el interés en profundizar y discutir los matices, las complejidades y los significados de la gestión menú y su componente educativo dentro de la actuación del nutricionista perteneciente a la red de AE en los municipios del Estado de San Pablo (11).
Durante el mes de enero de 2012, se realizó el mapeamento e identificación de los responsables técnicos a través de la página web de los departamentos de AE de la Secretaría de Educación del municipio de San Pablo y de los municipios mencionados. En la selección de los participantes se utilizó la técnica "bola de nieve" (snowball technique), método que permite la definición de una muestra por medio de referencias hechas por personas que comparten o conocen a otras y que poseen características de interés de la búsqueda (12). Se empezó a partir de una nutricionista matriculada en la Facultad de Salud Pública de la Universidad de San Pablo (USP) que actuaba en la $A E$ en uno de los municipios. El contacto con los nutricionistas representantes de las escuelas municipales se realizó por vía telefónica y/o electrónica, explicándoles los objetivos del estudio e invitándoles a participar voluntariamente.

Para la determinación del tamaño de muestra, se tuvo en cuenta que para los estudios cualitativos la variable crítica no es la cantidad de participantes y si la búsqueda intencional de variabilidad y calidad de las informaciones proporcionadas por los nutricionistas a ser entrevistados de acuerdo con su experiencia (13), aspecto evaluado según año graduación, donde se consideró como requisito mínimo tener seis meses, como tiempo suficiente para tener una base de actuación en el área, y máximo diez años de entrenamiento, al suponer que tiempos mayores pueden alterar los resultados en función a la diferencia curricular.

En la recolección de datos se utilizó grupo focal, técnica en la cual se obtienen datos a partir de reuniones en grupo con personas que representan el objeto de estudio, siendo una de las técnicas más usadas en los estudios cualitativos al involucrar la observación e interacción con los entrevistados y creando así múltiples líneas de comunicación (13-15). El proceso de desarrollo del grupo focal contiene procedimientos que buscan la comprensión de experiencias a partir de vivencias y puntos de vista de los participantes, sin que uno sea más importante que otro, pues la existencia de los mismos es la riqueza del estudio, es decir, todos los hallados tienen la misma relevancia. Una de sus mayores ventajas es que se basa en la tendencia humana de formar opiniones y actitudes durante la interacción entre los individuos, que no se logra con los cuestionarios cerrados o entrevistas individuales, porque a cada individuo se le invita a emitir opiniones sobre asuntos que, tal vez, nunca hayan pensado anteriormente (16).

Al contar con la oportunidad de trabajar con participantes de un nivel de compromiso suficiente con el área de AE se tuvo en cuenta que el número de participantes, según Aschidamini \& Saupe (17), oscilara entre un mínimo de seis y un máximo de doce personas.

El grupo focal se llevó a cabo con siete nutricionistas que aceptaron participar en el estudio, siendo dos representantes de São Bernardo, dos de Embu das Artes, una de Suzano y dos representantes de escuelas estatales ubicadas en la el municipio de San Pablo. Se realizó en la Facultad de Salud Pública de la USP y contó con la participación de una psicóloga con experiencia en la técnica para realizar el papel de mediadora. Fue elaborada una guía con el fin de dirigir la reunión y de mantener la línea del objetivo del estudio, para lo cual se abordaron preguntas referentes a la trayectoria profesional de los participantes, ejecución de actividades educativas en el ámbito de la AE y aspectos facilitadores o limitantes del desempeño de las actividades educativas.

El análisis del material obtenido tuvo como base la propuesta de Attride-Stirling (18), denominada redes temáticas. Esta propuesta analítica es una forma de organizar los datos 
cualitativos de acuerdo a los temas que surgen en diferentes niveles, lo que incluye seis pasos básicos: 1) codificación del material: reducción del material y ubicación de los segmentos textuales con significado relevante para el análisis; 2) identificación de los temas: destaque de los temas de interés y traslado para un nuevo documento; 3) construcción de redes temáticas: identificación de grandes grupos temáticos codificados con nombre y agrupados por temas semejantes; 4) descripción y exploración de las redes temáticas: constituye la primera parte del análisis, teniendo en cuenta que en la construcción de las redes se deben realizar varias lecturas para mejorar la comprensión y el significado de los temas; 5) resumen de los temas: elaboración de una síntesis con los un los temas principales y características que los componen; y 6) interpretación de las características encontradas.

Este estudio se realizó de acuerdo con las directrices y normas reguladoras de la Resolución CNS 196/96 del Consejo Nacional de Salud y aprobado por el Comité de Ética en Investigación de la Facultad de Salud Pública de la USP (proceso $\mathrm{n}^{\circ} 2287$ ).

\section{RESULTADOS}

La población de estudio estuvo conformada por siete nutricionistas, todas mujeres, graduadas a partir de 2001, con media de edad de treinta años y con experiencia laboral en $A E$ entre seis meses a diez años.

El grupo focal tuvo una duración de 1 hora y 16 minutos, el cual fue grabado en su totalidad y transcrito en 25 páginas. Después de la codificación del material, se obtuvo 16 páginas con los segmentos de texto que tenían significado relevante para el análisis, sumando 61 fragmentos de las narrativas de las nutricionistas. Dichos fragmentos fueron agrupados y dieron origen a 4 redes temáticas (tabla 1): actividades educativas realizadas y/o planificadas en la $A E$; enfrentamiento de las dificultades para el desarrollo de actividades educativas; elementos facilitadores para el desarrollo de actividades educativas; y contribución de la formación académica para la actuación en la $\mathrm{AE}$.

En la red temática denominada "actividades educativas realizadas y/o planificadas en la alimentación escolar», se recopilaron tres temas: realización de EAN con los alumnos, actividades de concientización con el equipo pedagógico e incorporación del tema alimentación y nutrición en los planes de estudio.

Al señalar las estrategias educativas planificadas, los participantes destacaron que no lograron llevarlas a cabo de la forma deseada, debido a la sobrecarga de trabajo en la AE. Estos informes dieron origen a la red temática "enfrentamiento de las dificultades para el desarrollo de actividades educativas". En este caso, se plantearon tres temas: "el elevado número de actividades que realizaban", "el reducido número de profesionales responsables" y "la deficiencia de conocimientos sobre los procedimientos más burocráticos sobre el menú", específicamente, los procesos involucrados en su gestión y planeación que demanda mucho tiempo y atención por parte del nutricionista, según se observa en los fragmentos a continuación: "Hay que hacer de todo un poco, hacer licitación, compras, menú, las cosas administrativas (...) si la nutricionista hiciera sólo las actividades educativas, podría facilitar un poco."; "Somos un equipo pequeño, pero estamos buscando más profesionales que se nos unan."; "Yo creo que estamos así también porque nos dedicamos poco, con el tema administrativo nosotros trabajamos bastante con lo administrativo, la licitación de compras y al final no tenemos una buena base en gestión pública, nos Ileva mucho tiempo (...)."

El análisis de la red temática además muestra que los nutricionistas a pesar de la gran preocupación por gestionar el menú, no reconocen su planeación como una estrategia educativa. Esto significa no reconocer su primer y principal instrumento de trabajo como educativo, situación observada anteriormente teniendo en cuenta que el proceso de planeación se identificó como una barrera y no como facilitador de la EAN.

Es comprensible y aceptable que los nutricionistas no se den cuenta de tal posibilidad, ya que existe una fuerte presión para evitar que hayan problemas con la administración del servicio de $A E$, específicamente que no falte comida, lo que no implica necesariamente calidad nutricional, como revela el siguiente testimonio: "La alimentación escolar es un área en el que la política, tanto la política como el partido son inherentes

\section{TABLA 1}

Redes temáticas sobre la gestión del menú por parte de nutricionistas

\section{Redes temáticas}

R1 Actividades educativas realizadas y/o planificadas en la AE.

R2 Enfrentamiento de las dificultades para el desarrollo de actividades educativas.

R3 Elementos facilitadores para el desarrollo de actividades educativas.

R4 Contribución de la formación académica para la actuación en la AE.

\section{Temas}

- Realización de EAN con los alumnos.

- Actividades de concientización con el equipo pedagógico.

- Incorporación del tema alimentación y nutrición en plan de estudios.

- El elevado número de actividades que eran realizadas.

- El reducido número de profesionales responsables.

- La deficiencia de conocimientos sobre los procedimientos más burocráticos sobre el menú.

- Acoplamiento del equipo pedagógico.

- Participación de los practicantes.

- Visión de Salud Pública, saber relacionar los problemas de la sociedad con la actuación profesional.

- Participación en proyectos de extensión.

- Prácticas y proyectos en comunidades con la participación de otros profesionales. 
en las actividades, todo lo que usted va hacer tiene que pensarlo dos veces, cuál va ser la consecuencia de eso que va a hacer, cierto?"; "Es un área de objetivo político porque incluye mucho dinero, da mucha visibilidad: mis niños están comiendo bien, hacen tal cosa. Que no siempre es lo más nutritivo, a veces uno dice que lo nutritivo sería una cosa, pero él quiere servir arroz y frijoles porque eso aquello es sobresaliente, da notoriedad, cierto? Da una buena imagen de mi gestión, mis niños están bien alimentados (...)."

La participación del equipo pedagógico es otro elemento facilitador del trabajo del nutricionista dentro del ambiente escolar. Esa facilidad va unida al hecho de que para incluir el aprendizaje en salud y nutrición como parte de la cultura del servicio de $A E$, se necesita del reconocimiento de toda la escuela para que la comunidad educativa se haga participe del proyecto y tome conciencia de su dimensión, así como se refiere en el siguiente párrafo: "Nosotros decimos que la dirección cambia todo dentro de una escuela, ahí usted se da cuenta cuando el director actúa, cuando realmente le interesa la alimentación, cuando él va a ir allá, al lado de la preparadora, estimula a los alumnos, cierto ?, es ahí cuando se hace el proyecto y está super involucrado, mejor dicho la escuela se moviliza y da gusto. Ahora, hay directores que ni saben que existe la alimentación allá en las cocina dentro de la escuela, cierto?"

\section{DISCUSIÓN}

Los temas abordados por las nutricionistas que participaron en el grupo focal son los mismos destacados en otros estudios como el de Iuliano (7), que señala la creación de huertas escolares, o de Schmitz et al. (19) que muestra la importancia de la formación de los profesores para que sean conscientes de su papel como multiplicadores de información sobre alimentación y nutrición. Pietruszynski et al. (20) discute la importancia de los alimentos en actividades que se desarrollan durante las clases, al contrario de actividades aisladas y que ocurren periódicamente. Pietruszynski et al., aparte de las acciones anteriormente mencionadas, destaca el alimento como un instrumento pedagógico y dice que la propuesta es una oportunidad de hacer que dicho conocimiento sea importante para el estudiante, pues es un elemento de la vida cotidiana, presente de forma obligatoria en todas las casas y afectado por diversas representaciones sociales y culturales.

Las narrativas referentes a las estrategias educativas planificadas coinciden con lo encontrado en el estudio de Assao et al. (21), el cual tuvo por objetivo identificar las percepciones vinculadas a la $A E$ entre el personal encargado de la cocina de las escuelas públicas del municipio de Guarulhos. En su discurso se identificaron observaciones sobre la calidad de alimentos como frijoles, carnes y verduras, que con frecuencia llegan a las escuelas en condiciones regulares, por lo que se refería que no había supervisión adecuada. Se sugirió que esto sucede porque los departamentos de AE no siempre cuentan con un talento humano de nutricionistas suficiente para atender tal demanda, aunque exista una planeación central con responsabilidad de la administración municipal o estadual.

El menú es responsabilidad técnica del profesional, así muchos estudios han llamado la atención sobre la importancia de ofrecerlo en forma adecuada para la formación de hábitos alimentarios saludables sobretodo en niños $(1,3,22)$. El nutricionista además de ser el administrador del servicio, tiene la oportunidad de desarrollar otros papeles, siendo también educador, al estar presente en la transformación del espacio de $A E$ en un ambiente de promoción de la salud y del apren- dizaje. La resolución del Consejo Federal de Nutricionistas (8) indica que en la elaboración de menús, el nutricionista deberá desarrollar actividades que irán desde el cálculo de parámetros nutricionales con base a recomendaciones nutricionales hasta el desarrollo proyectos de EAN para la comunidad escolar, e incluso la promoción de conciencia ecológica y medio ambiental. El Fondo Nacional para el Desarrollo de la Educación considera como estrategia de EAN la oferta de alimentación saludable en las escuelas (23).

El hecho de que el menú no se planee buscando su vertiente educacional puede generar situaciones de confusión a la hora de elegir los alimentos, de forma que la selección prioriza practicidad y bajo costo, y no la calidad de alimentos, sin favorecer la promoción del menú como estrategia para la EAN, lo que tampoco conlleva a la formación de hábitos alimentarios saludables, situación descrita por Assao et al. (21) que destaca que por causa de las limitaciones, principalmente de financiamiento, primaban los criterios de bajo precio para la compra de alimentos sobre las características nutricionales, calidad y aceptabilidad de los niños.

A partir de las narrativas sobre administración del servicio de $A E$ se puede verificar como el aspecto político puede ser una limitación en el trabajo de los nutricionistas que se desempeñan en la AE. Es evidente que falta, en la percepción de los gestores políticos y administrativos, el reconocimiento de que los escolares están la mayor parte del día en la escuela y que desde el punto de vista nutricional, no sólo tienen la oportunidad de ingerir alimentos para satisfacer sus necesidades biológicas, sino también la de compartir y adquirir hábitos alimentarios, cumpliendo la dimensión social de los programas de AE (1).

Frente a las dificultades mencionadas se percibe que el reconocimiento del menú como herramienta educativa depende de un trabajo intersectorial y no sólo del nutricionista como profesional responsable. Por lo tanto, es necesaria la concientización por parte de diferentes actores relacionados con la gestión de la $A E$, entre ellos la dirección de la escuela y el mismo gobierno, ya que para tal adecuación es preciso incluir intervenciones que se orienten a la planeación del menú, mejoras en la compra y preparación de los alimentos, promoción de elecciones más saludables, formación y apoyo permanente a los preparadores de la AE (24).

El gobierno necesita reconocer la importancia de la oferta de una alimentación equilibrada y nutricionalmente adecuada que contribuya a la EAN de los estudiantes, además de verificar la incoherencia entre el presupuesto y la cobertura de las necesidades de los escolares en términos de calidad nutricional, pues el escenario epidemiológico revela la importancia de tomar medidas al respecto. Es así como el ranking elaborado por la Organización para la Cooperación y Desarrollo Económico (25) refiere que a nivel mundial Chile es el sexto país con más obesidad infantil y Brasil el décimo quinto; en latinoamérica, el primero es Chile, seguido por Brasil. Chile presenta un aumento de obesidad infantil que ha llegado a ser un importante problema de salud pública y de nutrición, ya que aumenta cada año como lo destaca Vio del R. et al. (26). Las medidas tomadas frente al combate de la obesidad en Chile se han direccionado a acciones de gobierno que consideran necesario modificar el programa de $A E$, siendo uno de sus desafíos entregar una dieta saludable para contribuir en la disminución de obesidad (27). Lo mismo sucede con Brasil, como se recoge en la Orden Interministerial 1010 (28), los cambios en el perfil epidemiológico de la población con énfasis en el exceso de peso y obesidad, especialmente entre niños 
y adolescentes, ocasionado por el modelo de alimentación occidental contemporánea que presenta la predominio de una alimentación densamente calórica, rica en azúcar y grasa animal además de ser reducida en carbohidratos complejos y fibras, además de ser reconocido el gran desafío de incorporar el tema de la alimentación y la nutrición en el contexto escolar, haciendo hincapié en la alimentación saludable y en la promoción de la salud.

La importancia de tales acciones se confirma por Kac \& Velásquez-Meléndez (2) cuando reflexiona acerca del papel que hoy ejerce la obesidad en el complejo perfil epidemiológico de las poblaciones latinoamericanas, teniendo en cuenta que los estudios confirman la creciente magnitud de la obesidad en niños y adolescentes condicionada a determinantes del estilo de vida sedentario y consumo de dietas no adecuadas, pero sobre todo, por la falta de una mayor diversidad en las intervenciones y apoyo gubernamental con la implementación de acciones claras de prevención y lucha contra la obesidad.

Sobre la oferta se percibe que ya existe una concepción adecuada por parte del gobierno brasileño, dado que definió acciones para alcanzar una alimentación saludable en el entorno escolar, entre las cuales se incluye: la definición de estrategias junto con la comunidad escolar para favorecer elecciones saludables; restricción de alimentos con un alto contenido de grasa, grasas saturadas, grasas trans, bajos en azúcar y sal; desarrollo de opciones de alimentos y comidas saludables; aumento de la oferta y promoción del consumo de frutas y verduras; estimulo y colaboración a los servicios de alimentos de la escuela para la divulgación de opciones saludables y desarrollo de estrategias que posibiliten esas elecciones (28). La asignación monetaria de la AE en Brasil para estados y municipios es de US\$ 0,15 estudiante/día por un tiempo de comida principal (almuerzo) y una tiempo intermedio (onces) en la educación primaria y secundaria. El trabajo realizado por Goldberg et al. (24) encontró que la inclusión de alimentos más saludables y promoción de hábitos adecuados relacionados con el mejoramiento del menú, sólo el gasto en productos frescos representó un aumento de US\$ 27 mil en relación al año anterior, sin embargo esas y otras intervenciones correspondientes a la calidad del menú contribuyó en el incremento de la participación de los estudiantes en la AE y mejoría del perfil nutricional. Las presiones financieras son reconocidas como las principales barreras para la preparación de comidas más nutritivas (22).

En consonancia a la participación de la comunidad educativa, Abreu (29) señala que la ausencia de una propuesta clara de trabajo, formulada y coordinada por los órganos centrales de administración educativa, se relaciona con la manera en que la AE pasa a depender en gran parte de la concepción de iniciativa y hasta de buena voluntad de aquellos que son responsables de su ejecución. El rendimiento de las nutricionistas, de los profesores en cargos directivos y de los funcionarios de la cocina es primordial en la definición del sentido y del papel de la alimentación en las unidades escolares.

En el estudio de intervención realizado por Pearlman et al. (30) en escuelas públicas de Estados Unidos se encontró que para el desarrollo de comportamientos alimentarios adecuados, en tres de las cuatro escuelas estudiadas, el apoyo del director fue un componente clave para el éxito de la intervención.

La alianza entre escuela, políticos, padres y comunidad debería trabajar en la creación de un ambiente donde los niños puedan comer de forma saludable y desarrollen hábitos que contribuyan al bienestar a lo largo de su vida, ya que los hábi- tos alimentarios adquiridos durante la infancia, se mantienen en la adolescencia y perduran hasta la edad adulta. De esta forma se estarían efectuando esfuerzos para la prevención de la obesidad $(22,31)$.

De acuerdo con Story et al. (22), debe dedicarse más voluntad y recursos a la política e implementación a nivel federal, estatal y local. Igualmente es necesario el financiamiento en investigación para crear y evaluar las acciones de prevención de obesidad, innovación de intervenciones en las escuelas por medio de estrategias comportamentales y ambientales, una modificación en las estrategias políticas. Para hacer esto, se necesita de un plan sistemático y de voluntad política que coloque como prioridad la salud y el bienestar de los escolares.

\section{CONCLUSIONES}

La percepción de la gestión menú como una responsabilidad administrativa del nutricionista se sobrepone a su función educativa, dejando de un lado y siendo invisible tanto para el profesional como también para los otros actores de la escuela.

La calidad de la alimentación y por ende, nutricional en el ámbito de la AE es un compromiso social, incluso teniendo restricciones presupuestales, y para ello es imprescindible el reconocimiento y trabajo colectivo de lo que comunica el menú, involucrando no sólo a los nutricionistas responsables, sino también a los profesores, a la comunidad, gestores de las escuelas y de gobierno.

La implementación de estrategias educativas en alimentación y nutrición es prioritaria para los escolares. Una de las formas es la planeación del menú, buscando no sólo cubrir las necesidades biológicas y nutricionales, sino también como instrumento para la EAN, ya que el refuerzo de los hábitos alimentarios es influenciado por el ambiente escolar.

Es indiscutible la importancia y necesidad de la transformación del espacio escolar en un ambiente de promoción de la salud, una vez que la promoción de la familiarización con los alimentos y el incentivo a su consumo adecuado, posibilitan la formación de individuos críticos y con hábitos de vida saludables, contribuyendo a la prevención de la obesidad y de enfermedades crónicas relacionadas.

\section{RESUMEN}

El debate sobre alimentación escolar (AE) y su impacto en el perfil epidemiológico exige la formulación de acciones reguladoras y con planeación educativa. El presente trabajo tuvo como objetivo discutir la gestión del menú y su reconocimiento en la vertiente educacional de la $A E$. Fue un estudio cualitativo con participación de nutricionistas que actúan en AE de municipios vecinos de la Región Metropolitana de San Pablo, cuya recolección de datos usó la técnica del grupo focal y para el análisis siguió la propuesta de redes temáticas. Al momento de señalar las estrategias educativas planeadas, los participantes destacaron no haberlas llevado a cabo de la forma deseada debido a la sobrecarga de trabajo y a pesar de la gran preocupación que la gestión del menú generó, esta no fue contemplada como estrategia educativa. La interpretación de educación puede ser reconocida en acciones formales y explicitas, sin embargo la gestión del menú trasciende los canales tradicionales de comunicación, al enfocarse en el consumo como momento de aprendizaje y promoción de hábitos de alimentación saludable, contribuyendo y reforzando en la prevención de obesidad dentro del ambiente escolar.

Palabras clave: Alimentación escolar; menú pedagógico; programas nutricionales; escuelas promotoras de la salud; obesidad. 


\section{BIBLIOGRAFÍA}

1. Pino V. J L, López E. M Á, Cofré T. M I, González R. C, Reyes C L. Conocimientos alimentario-nutricionales y estado nutricional de estudiantes de cuarto año básico según establecimientos particulares y subvencionados de la ciudad de talca, Rev Chil Nutr 2010; 37 (4): 418-26.

2. Kac $G$, Velásquez-Meléndez $G$. A transição nutricional e a epidemiologia da obesidade na América Latina, Cad. Saúde Pública, Rio de Janeiro 2003; 19 (Sup. 1): S4-S5.

3. Crovetto M. M, Zamorano F. N, Medinelli S. A. Estado nutricional, conocimientos y conductas en escolares de kinder y primer año básico en 3 escuelas focalizadas por obesidad infantil en la comuna de Valparaíso, Chile. Rev Chil Nutr. 2010; 37 (3): 309-20.

4. Fundo Nacional de Desenvolvimento da Educação - FNDE. Programas: Alimentação escolar. FNDE. Disponible en: http://www.fnde.gov.br/programas/alimentacao-escolar/ alimentacao-escolar-apresentacao. 2012.

5. Salinas C. J, Vio del R. F. Programas de salud y nutrición sin política de estado: el caso de la promoción de salud escolar en Chile, Rev Chil Nutr. 2011; 38 (2): 100-16.

6. Iuliano, B. A. Atividades para promoção de alimentação saudável em escolas de ensino fundamental do município de Guarulhos - SP. 2008. Dissertação (Mestrado em Saúde Pública) - Faculdade de Saúde Pública, Universidade de São Paulo, São Paulo, 2008.

7. Iuliano, B. A, Cervato-Mancuso, A. M, Gambardella, A. M. $D$. Educação nutricional em escolas de ensino fundamental do município de Guarulhos-SP, O Mundo da Saúde, São Paulo 2009; 33 (3): 264-72.

8. Conselho Federal de Nutricionistas. Dispõe sobre as atribuições do Nutricionista no âmbito do Programa de Alimentação Escolar (PAE) e dá outras providências, Resolução CFN $n^{\circ}$ 358, de 18 de maio de 2005.

9. BRASIL. Lei $n^{\circ} 1.947$, de 16 de junho de 2009. Dispõe sobre o atendimento da alimentação escolar e do Programa Dinheiro Direto na Escola aos alunos da educação básica; e dá outras providências, Diário Oficial da União, Brasília, DF, 17 jun. 2009.

10. Secretaria da Educação do Estado de São Paulo. Disponible en: http://escola.edunet.sp.gov.br/pesquisas/index_escolas. asp?cod_mun $=100 \&$ Ensino $=0 \&$ Modalidade $=0 \&$ Modalida de_Det=0\&rede=0\&nome.

11. Manson J. Qualitative researching. SAGE publication Ltd, London, 2006.

12. Biernacki P., Waldford D. Snowball sampling: problems and techniques of chain referral sampling. Sociol Methods Res. 1981; 10: 141-63.

13. Denzin N.K, Lincoln YS. Handbook of qualitative research. SAGE publication Ltd, Thousand Oaks, 2000.

14. Domper A, Zacarías H.I, Olivares C. S, Hertrampf D. E. Evaluation of a nutrition information program. Rev Chil Nutr. [revista en la Internet]. 2003 Abr [citado 2013 Ene 18] ; 30(1): 43-51.

15. Minneman Rebecca M, Hennink Monique M, Nicholls, Andrea et al., "Barriers to Testing and Treatment for Chagas Disease among Latino Immigrants in Georgia," Journal of Parasitology Research, Article ID 295034, 11 pages, 2012. doi:10.1155/2012/295034.

16. Iervolino AS, Pelicioni MCF. A utilização do grupo focal como metodologia qualitativa na promoção da saúde, Rev. Esc Enf USP 2001; 35 (2): 115-21.
17. Aschidamini I. M, Saupe R. Grupo focal: estratégia metodológica qualitativa: um ensaio teórico, Cogitare Enferm 2004; 9: 9-14.

18. Attride-Stirling J. Thematic networks: an analytic tool for qualitative research, Qualitative Res. 2001; 1 (3): 385-405.

19. Schmitz B. A. S, Recine E, Cardoso G. T, Silva J. R. M, Amorim N. F. A, Bernardon R, Rodrigues M. L. C. F. A escola promovendo hábitos alimentares saudáveis: uma proposta metodológica de capacitação para educadores e donos de cantina escolar, Cad. Saúde Pública, Rio de Janeiro, 2008; 24 (2): S312 - S22.

20. Pietruszynski E. B, Albiero K. A, Pöpper G, Teixeira P. F. Práticas pedagógicas envolvendo a alimentação no ambiente escolar: apresentação de uma proposta, Rev Teoria Prática Educação, 2010; 13 (2): 223-9.

21. Assao T. Y, Westphal M. F, Bógus C. M, Cervato-Mancuso A. M. Alimentação do escolar: percepção de quem prepara e oferece as refeições na escola, Segurança Alimentar Nutr. (Campinas) 2012; 19 (1): 22-32.

22. Story M, Nanney MS, Schwartz MB. Schools and Obesity Prevention: Creating School Environments and Policies to Promote Healthy Eating and Physical Activity. Milbank Quarterly 2009; 87 (1): 71-100.

23. BRASIL. Resolução/CD/FNDE - n.38, de 16 de Julho de 2009. Dispõe sobre o atendimento da alimentação escolar aos alunos da educação básica no Programa Nacional de Alimentação Escolar - PNAE. 2009.

24. Goldberg JP, Collins JJ, Folta SC, McLarney MJ, Kozower C, Kuder J, et al. Retooling Food Service for Early School Students in Somerville, Massachusetts: The Shape Up Somerville Experience, Preventing Chronic Disease. Public Health Res, Practice, Police 2009; 6 (3): 1-8.

25. La Tercera. Ocde: Chile es el sexto país con más obesidad infantil. Disponible en: http://diario.latercera. com/2012/02/24/01/contenido/pais/31-101553-9-ocdechile-es-el-sexto-pais-con-mas-obesidad-infantil.shtml. 2012, p.14

26. Vio del F. R, Zacarías H. I, Lera L, Benavides M. C, Gutiérrez A. M. Prevención de la obesidad en escuelas básicas de Peñalolén: componente alimentación y nutrición, Rev Chil Nutr. 2011; 38 (3): 268-76.

27. Chile. Junaeb - Junta Nacional de Auxilio Escolar y Becas. Ministério de Educación. Disponible en: http:// www.junaeb.cl/prontus_junaeb/site/artic/20120306/ pags/20120306131003.html

28. BRASIL. PORTARIA INTERMINISTERIAL N 1.010 DE 8 DE MAIO DE 2006. Institui as diretrizes para a Promoção da Alimentação Saudável nas Escolas de educação infantil, fundamental e nível médio das redes públicas e privadas, em âmbito nacional. 2006.

29. Abreu M. Alimentação escolar: combate à desnutrição e ao fracasso escolar ou direito da criança e ato pedagógico? Em Aberto, Brasília, ano 15, n.67, jul./set. 1995.

30. Pearlman D. N, Dowling E, Bayuk C, Cullinen K, Thacher A. K. From Concept to Practice: Using the School Health Index to Create Healthy School Environments in Rhode Island Elementary Schools, Preventing Chronic Disease. Public Health Res, Practice, Police 2005; 2: 1-16.

31. Bravo A. M, Martín N. U, González A. G. Evaluación de los hábitos alimentarios de una población de estudiantes universitarios en relación com sus conocimientos nutricionales, Nutr Hosp. 2006;21(4):466-73. 